Int. J.Morphol.,

39(3):915-919, 2021.

\title{
Disposición Infrecuente de la Arteria Digital Palmar Propia Radial del Índice
}

\author{
Uncommon Disposition of the Index Radial Proper Palmar Digital Artery
}

Homero F. Bianchi ${ }^{1,2}$ \& Nicolás E. Ottone ${ }^{3,4,5}$

\begin{abstract}
BIANCHI, H. F. \& OTTONE, N. E. Disposición infrecuente de la arteria digital palmar propia radial del índice. Int. J. Morphol., 39(3):915919, 2021.

RESUMEN: Se presentan 3 casos de variación de origen de la arteria digital palmar propia radial del índice, dos de los cuales tienen su origen en la rama palmar superficial de la arteria radial, no detalladas previamente en la literatura, junto a variaciones vasculares en uno de ellos, mientras que en el tercero, su origen en la arteria digital palmar propia ulnar del pulgar, es fuente de controversia. Esto es discutida en la presente comunicación. Se describen anastomosis vasculares entre la arteria ulnar superficial, la rama palmar superficial de la arteria radial, la primera arteria interósea dorsal y la arteria digital palmar propia radial del índice, como circuitos anastomóticos alternativos.
\end{abstract}

PALABRAS CLAVE: Arteria digital palmar propia radial del índice; Circuitos anastomóticos vasculares palmares; Variaciones anatómicas.

\section{INTRODUCCIÓN}

La arteria digital palmar propia radial del índice (ADPPRI) es descripta con diversos orígenes: arco palmar superficial (APS) (Dubreuil-Chambardel, 1926; Al-Turk \& Metcalf, 1984), primera arteria metacarpiana (1AMTC) (AlTurk \& Metcalf; Whaterby), la arteria digital palmar propia ulnar del pulgar (ADPPUP) (Murakami et al., 1969; Tasom et al., 2014) o del arco palmar profundo (Delorme, 1882). Earley (1986) señala su origen en la rama palmar superficial de la arteria radial (RPSAR), o de acuerdo con Ilic et al. (2018), cuando la 1AMTC, alcanzando un desarrollo considerable, emite las ramas colaterales para el pulgar y el índice. La disección de tres preparados de miembro superior, llevada a cabo en nuestro laboratorio, dentro de la búsqueda de variaciones anatómicas, mostro tres ADPPRI con disposiciones infrecuentes.

\section{MATERIAL Y MÉTODO}

La muestra consiste en tres miembros superiores, provenientes de adultos mayores, dos de ellos correspondien- tes al mismo cadáver. En este cadáver, los dos miembros superiores fueron inyectados en estado fresco, sin fijación, con material elastomérico coloreado (rojo), para posteriormente ser fijados con formalina al 15\% durante 2 meses. Luego fueron sumergidos en peróxido de hidrógeno al 10\% durante 1 semana. El restante miembro superior, correspondió a material disecado previamente destinado a material de prosección para estudiantes, se encontraba fijado y sumergido en formalina al $15 \%$. La disección de los tres miembros superiores se realizó con técnica habitual, empleando instrumental de disección estándar y de microcirugía, y lupas de magnificación de $2 \mathrm{x}$.

\section{RESULTADOS}

Preparado 1, lado izquierdo (Figs. 1A-F). La arteria radial (AR), próxima a la muñeca, emite la rama palmar superficial de la arteria radial (RPSAR), la cual, $20 \mathrm{~mm}$ proximal a la articulación metacarpofalángica del pulgar, emite la ADPPRI, la cual pasa debajo de los nervios digitales

\footnotetext{
${ }^{1}$ Cirujano de Mano, Profesor Consulto, Departamento de Anatomía, Facultad de Medicina, Universidad de Buenos Aires, Buenos Aires, Argentina. ${ }^{2}$ Departamento de Anatomía, Fundación Barceló, Instituto Universitario de Ciencias de la Salud, Buenos Aires, Argentina.

${ }^{3}$ Laboratorio de Plastinación y Técnicas Anatómicas, Centro de Investigación en Ciencias Odontológicas (CICO), Facultad de Odontología, Universidad de La Frontera, Temuco, Chile.

${ }^{4}$ Departamento Odontología Integral Adultos, Facultad de Odontología, Universidad de La Frontera, Temuco, Chile.

${ }^{5}$ Centro de Excelencia en Estudios Morfológicos y Quirúrgicos (CEMyQ), Facultad de Medicina, Universidad de La Frontera, Temuco, Chile.
} 
palmares propios del pulgar, continuando como arteria digital palmar propia radial del pulgar (ADPPRP) (Figs. 1A,B). Sobre el margen medial de la eminencia tenar, y proximal a la ADPPRI, se observa una arteria anastomótica con un ramo de la arteria ulnar superficial (AUS) (Figs. 1B,C). Al reclinar el margen medial de la masa muscular tenar (Fig. 1B), se aprecia que la ADPPRI deja de ser superficial y pasa debajo del tendón del m. flexor pollicis longus (FPL) (Fig. 1B). Debajo del fascículo profundo del $\mathrm{m}$. flexor pollicis brevis (FPB) y del FPL (Fig. 1B), emerge la arteria anastomótica con la AUS (AAUS), (Fig. 1C) pasando en su trayecto, debajo del nervio digital palmar propio ulnar del pulgar (Fig. 1c). La primera arteria interósea dorsal (1AIOD) (Fig. 1D) se divide en dos ramas, una medial, la arteria digital dorsal propia medial del pulgar (ADDPMP), y una lateral, que se divide en la AAUS y en la arteria digital palmar propia ulnar del pulgar (ADPPUP) (Figs. 1D,E). La primera, como se mencionó, emerge debajo del FPB y encima del margen superior del aductor pollicis (ADP), ubicándose luego sobre su cara anterior (Fig. 1E). La segunda se origina próxima a la articulación metacarpofalángica del pulgar (AMCFP), pasa debajo de la placa volar, perfora la aponeurosis extensora y se ubica en el lado medial del pulgar (Figs. 1E,F). El arco palmar superficial (APS) es de tipo incompleto, formado por la arteria ulnar superficial (AUS), de trayecto corto y transversal, no pasando el nivel del tendón flexor del dedo medio, suministrando la arteria medial del quinto dedo y las tres arterias digitales palmares comunes (ADPC) de los espacios interóseos palmares $2^{\circ}, 3^{\circ}, \mathrm{y} 4^{\circ}$ (Fig. 1A).
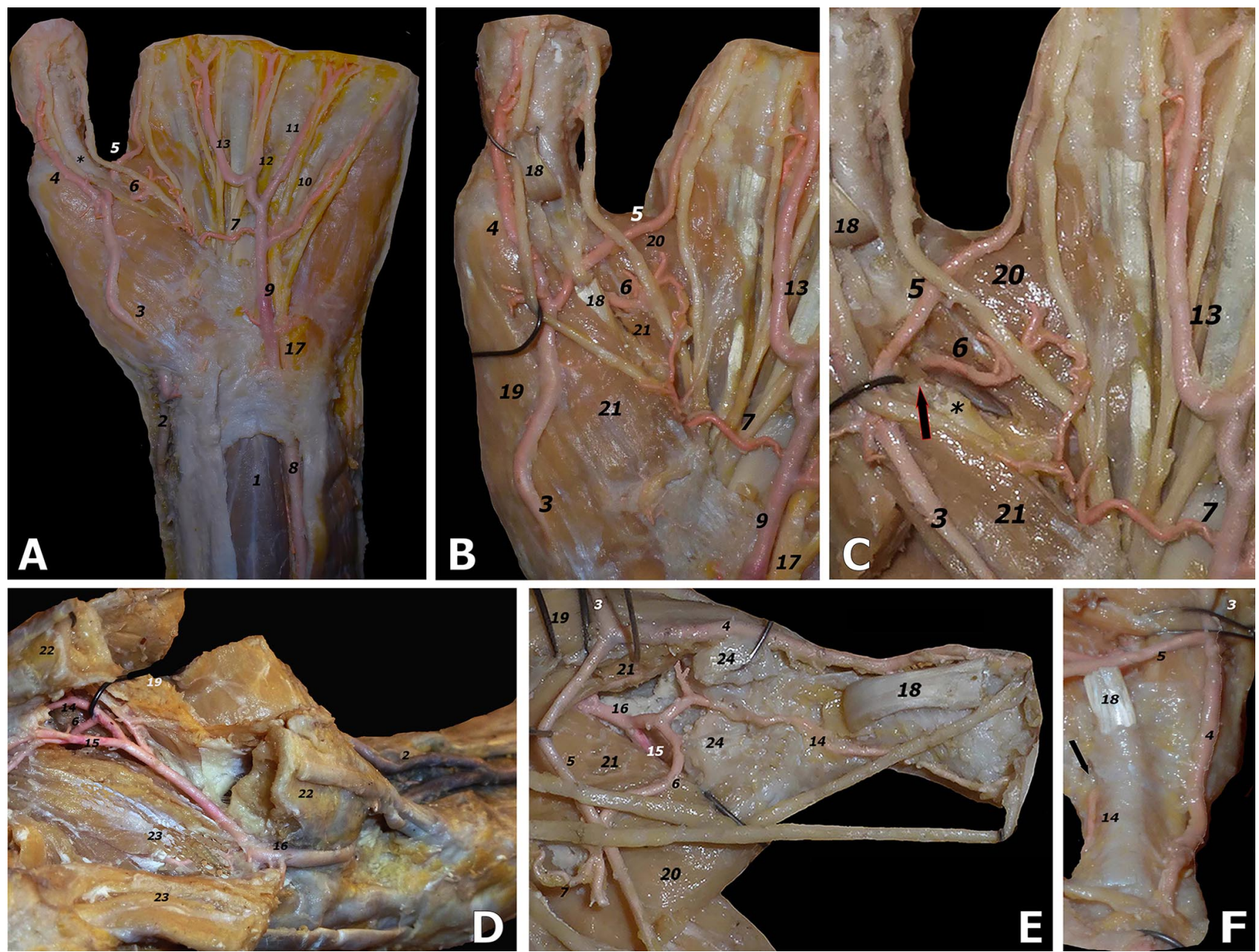

Fig. 1. Disección correspondiente al preparado 1, lado izquierdo. 1, m. flexor digitorum superficialis; 2, Arteria radial; 3, Rama palmar superficial de la arteria radial; 4, Arteria digital palmar propia radial del pulgar; 5, Arteria digital palmar propia radial del índice; 6-7, Anastomosis entre rama palmar superficial de la arteria radial y arteria ulnar superficial; 8, Arteria ulnar; 9, Arteria ulnar superficial; 1013, Arterias digitales palmares comunes de los espacios $4^{\circ}, 3^{\circ}, 2^{\circ}, 1^{\circ} ; 14$, Arteria digital palmar propia ulnar del pulgar; 15, Arteria digital dorsal propia medial del pulgar; $16,1^{\circ}$ arteria interósea dorsal; 17, Nervio ulnar; 18, tendón/vaina del m. flexor pollicis longus; 19, m. abductor pollicis brevis; 20, m. adductor pollicis; 21, m. flexor pollicis brevis; 22, $1^{\circ}$ metacarpiano; 23, Primer músculo interóseo dorsal; 24 , placa volar. 

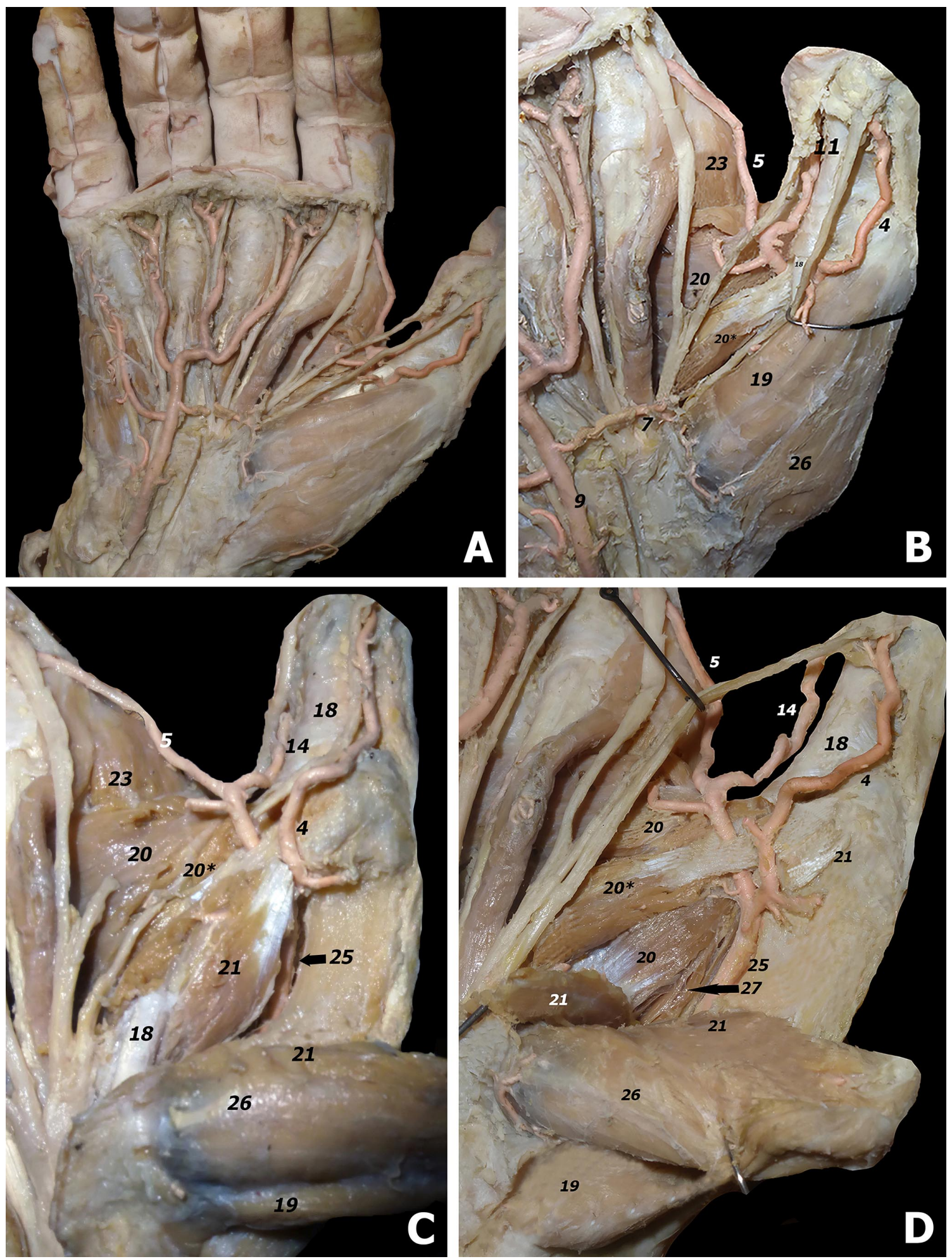

Fig. 2. Disección correspondiente al preparado 1, lado derecho. 4, Arteria digital palmar propia radial del pulgar; 5 , Arteria digital palmar propia radial del índice; 7, Anastomosis entre rama palmar superficial de la arteria radial y arteria ulnar superficial; 9, Arteria ulnar superficial; 10-13, Arterias digitales palmares comunes de los espacios $4^{\circ}, 3^{\circ}, 2^{\circ}, 1^{\circ} ; 14$, Arteria digital palmar propia ulnar del pulgar; 18, tendón/vaina del m. flexor pollicis longus; $19, \mathrm{~m}$. abductor pollicis brevis; 20,20* m. adductor pollicis; 21, m. flexor pollicis brevis; 23, Primer músculo interóseo dorsal; 25, Primera arteria metacarpiana; 26, m. opponens pollicis; 27, m. primer interóseo palmar. 
Preparado 1, lado derecho (Figs. 2A-D). La 1AMTC originada en la AR, muestra un trayecto habitual, por debajo del FPB, bordeando la cara medial del primer metacarpiano (Fig. 2C); llegada a nivel de la AMCFP, se dirige hacia adentro pasando debajo del FPL, de los fascículos de inserción del FPB (Fig. 2C), y de un fascículo accesorio del ADP, que termina debajo del FPL y sobre su vaina tendinosa (Figs. 2C,D). Emite la ADPPRP y continua como ADPPUP, de la cual se origina la ADPPRI (Figs. 2C,D), Se observa una anastomosis entre AR y AUS (Fig. 2A,B). El APS es similar al descripto en el espécimen del lado izquierdo. Las Figuras 2A,B muestra la vista del ejemplar, previo a la retracción muscular y visualización de las estructuras anteriormente mencionadas.

Preparado 2, lado izquierdo (Fig. 3). La RPSAR se divide, $17 \mathrm{~mm}$ proximal a la articulación MCFP, en ADPPRP y ADPPRI. A $10 \mathrm{~mm}$ del origen de la última, nace la AAUS, que sigue, pero a corta distancia el margen medial de la eminencia tenar. El APS emite la arteria medial del quinto dedo y la arteria digital palmar común del cuarto espacio y termina dividiéndose en dos ramas que son las arterias digitales palmares comunes de los espacios interóseos $3^{\circ}$ y $2^{\circ}$ (Fig. 3).

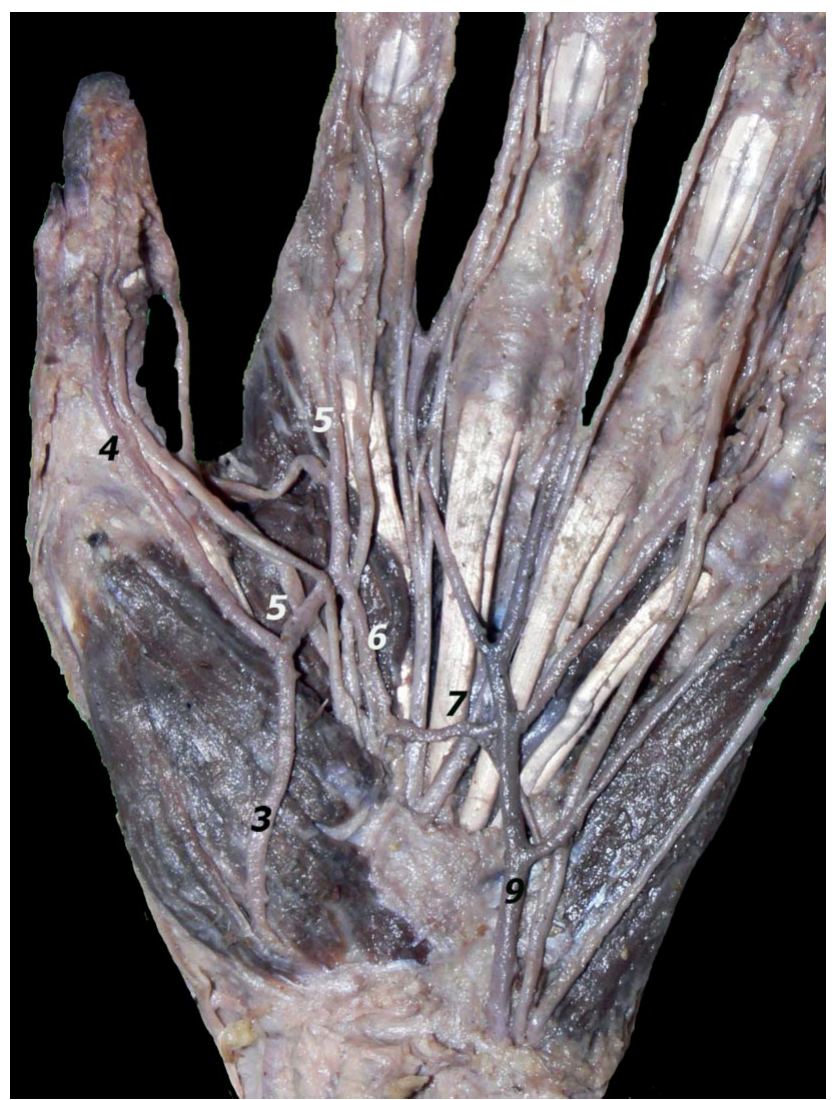

Fig. 3. Disección correspondiente al preparado 2, lado izquierdo. 3, Rama palmar superficial de la arteria radial; 4, Arteria digital palmar propia radial del pulgar; 5 , Arteria digital palmar propia radial del índice; 6-7, Anastomosis entre rama palmar superficial de la arteria radial y arteria ulnar superficial; 9, Arteria ulnar superficial.

\section{DISCUSIÓN}

Los preparados muestran orígenes de la ADPPRI diferentes de lo habitual, dos de ellos similares, en cuanto al origen, pero con características propias. En el espécimen número 1 , lado derecho, la arteria toma origen en la ADPPUP, origen señalado previamente por Poirier y Danish, citados en el tratado de Dujarier (1905), aunque éste lo considera raro y se inclina por la idea de Farabeuff, quien la asume como rama de la 1AMTC junto con las arterias del pulgar. Tasom et al. comparten nuestra idea en uno de sus preparados. Earley considera a la ADPPUP como rama terminal de la 1AMTC, lo que se observa en la mitad de sus casos. Como es admisible la dificultad de establecer en este caso el origen de la ADPPRI, la consideración de la arteria ulnar del pulgar como terminal validará a la ADPPRI como colateral (Figs. 2C,D). En una comunicación previa (Bianchi \& Leiro, 1987), se estableció que la 1AMTC, admitía más de un tipo de división, y entre ellas, la próxima a la primera comisura, en ambas ADPP del pulgar y la ADPPRI, era la menos frecuente, $2,5 \%$, motivo por el cual consideramos a la presente, como una variedad de división de esta última con origen de la ADPPRI en la ADPPUP. El trayecto de la 1AMTC es parecido al citado por Earley en relación con la posición debajo del tendón de inserción del FPB, del FPL y su vaina, y del fascículo accesorio del ADP, previo a la inserción en la vaina del FPL, quedando por delante de las inserciones de los manojos del ADP en el pulgar (Figs. 2C,D). La 1AMTC pertenece a la variedad pre-aductora (Earley; Bianchi \& Leiro) por la presencia del fascículo accesorio del AP, que interpretamos, es el que Williams (1998) denominó primer interóseo palmar (Fig. 2D).

El espécimen 1 lado izquierdo, y el espécimen 2, tienen aspectos comunes y diferentes. Entre los primeros, observamos la presencia de la RPSAR que se continúa como ADPPRP, después de dar origen a la ADPPRI a nivel de la articulación MCFP. Si bien este origen es mencionado por Earley, no da precisiones sobre su origen, trayecto y anastomosis. Entre las diferencias, mientras que en el espécimen 1 lado izquierdo nace de la RPSAR, con trayecto debajo del FPL, en el espécimen 2, con el mismo origen, pasa a la palma de la mano para seguir a su destino. En los tres casos, se observa un ramo anastomótico con la AUS, pero mientras que en el preparado 1 lado izquierdo es un ramo de la 1AIOD, en el preparado 1 lado derecho lo es de la RPSAR a nivel de la muñeca, naciendo en el espécimen 2 de la ADPPRI. La única cita es la de Tagil et al. (2012), similar a la de nuestro espécimen 1 lado derecho. Estas anastomosis constituyen otras formas de enlace entre los sistemas ulnar y radial, complementos del APS. 
El espécimen 1 lado izquierdo, muestra una variedad arterial con un trayecto nunca visto por nosotros, y no encontrado en la literatura consultada: el origen de la ADPPUP en la 1AIOD, de trayecto profundo, pasando debajo de la placa volar metacarpofalángica del pulgar, haciéndose superficial después de perforar la cincha aponeurótica dorsal (Zancolli \& Cozzi, 1993), expansión del tendón extensor, o dosel (Netter, 2006) de dicha articulación. En los tres preparados, el APS resultó de tipo incompleto, formado a expensas de la AUS, transverso y corto en el espécimen 1 ambos lados, mientras que en el preparado 2, el APS se bifurca en las ADPC de los espacios tercero y segundo (Bianchi \& Ottone, 2021).

La importancia de la variación de origen de la ADPPRI, radica que la arteria de origen, la RPSAR es empleada en cirugía plástica, ya sea como pedículo arterial en reimplantes de pulgar (Zheng et al., 2015), en colgajos libres o en aquellos a pedículo proximal, para cubrir defectos cutáneos de los dedos o de la eminencia tenar (Omokawa et al., 2002). Ese empleo se basa en la consideración de una disposición constante (Zheng et al.), lo cual, en nuestros casos, no se ha comprobado. De acuerdo a las técnicas descriptas, es posible que la disposición de la arteria como la describimos dificulte o no haga posible su aplicación, y que en un caso como los que describimos, la toma de la RPSAR podría, en teoría, producir una disminución del flujo sanguíneo del pulgar e índice, en especial en lesiones graves del pulgar, en relación con las características del lecho vascular, debiendo la variación de la ADPPUP ser considerada en la reparación de daños del pulgar.

\section{CONCLUSIONES}

Los casos descriptos comprueban que la disposición de la RPSAR no es constante, y que si bien su frecuencia es baja, la asociación con otras variedades vasculares, como el origen de la ADPPRI, puede tener implicancias desfavorables en técnicas de cirugía plástica reparadora, así como su origen en la ADPPUP, que debería considerarse dentro de las variaciones de división de la 1AMTC.

BIANCHI, H. F. \& OTTONE, N. E. Uncommon disposition of the index radial proper palmar digital artery. Int.J. Morphol., 39(3):915-919, 2021.

SUMMARY: We present 3 cases of variation of origin of the index radial proper palmar digital artery, two of which have their origin in the superficial palmar branch of the radial artery, not previously detailed in the literature, together with vascular variations in one of them, while in the third, its origin in the palmar proper ulnar digital artery of thumb, is a source of controversy. This is discussed in this communication. Vascular anastomoses between the superficial ulnar artery, the superficial palmar branch of the radial artery, the first dorsal interosseous artery, and the radial index palmar digital artery are described as alternative anastomotic circuits.

KEY WORDS: Radial palmar digital artery of the index; Palmar vascular anastomotic circuits; Anatomical variations.

\section{REFERENCIAS BIBLIOGRÁFICAS}

Al-Turk, M. \& Metcalf, W. K. A study of the superficial palmar arteries using the Doppler Ultrasonic Flowmeter. J. Anat., 138(Pt. 1):27-32, 1984.

Bianchi, H.F. \& Leiro, R. The arterial trunk of the thumb-index digital collaterals. Surg. Radiol. Anat., 9(1):63-7, 1987.

Bianchi, H. F. \& Ottone, N. E. Morphological aspects of the surface palmar arch in old adult individuals. Int. J. Morphol., 39(2):347-54, 2021.

Delorme, E. De la Ligature de la Paume de la Main et des Arteres de la Plante du Pied. Paris, Masson, 1882.

Dubreuil-Chambardel,L. Traité des Variations du Système Artériel. Paris, Masson, 1926.

Dujarier, C. Anatomie des Membres. Paris, Steinhel, 1905.

Earley, M. J. The arterial supply of the thumb, first web and index finger and its surgical application. J. Hand Surg. Br., 11(2):163-74, 1986.

Ilic, M.; Milisavljevic, M.; Malikovic, A.; Laketic, D.; Eric, D.; Boljanovic, J.; Dozic, A.; Stimec, B. V. \& Manojlovic, R. The superficial palmar branch of the radial artery: a corrosion cast study. Folia Morphol. (Warsz), 77(4):649$55,2018$.

Murakami, T.; Takaya, K. \& Outi, H. The origin, course and distribution of arteries to the thumb, with special reference to the so-called A. princeps pollicis. Okajimas Folia Anat. Jpn., 46(2):123-37, 1969.

Netter, F. H. Atlas de Anatomía Humana. $3^{\mathrm{a}}$ ed. Barcelona, Barron's, 2006.

Omokawa, S.; Tanaka, Y.; Ryu, J.; Suzuki, J. \& Kish, V. L. Anatomy of the ulnar artery as it relates to the transverse carpal ligament. J. Hand Surg. (Am), 27:101-4, 2002.

Tagil, S. M.; Bozkurt, M. C.; Kuvat, S. V. \& Cicekcibasi, A. E. A variant course of the superficial palmar branch of the radial artery. Surg. Radiol. Anat., 34(9):871-3, 2012.

Tasom, A.; Sinthubua, A.; Sananpanich, K. \& Mahakkanukrauh, P. Cadaveric study of superficial palmar arch variations in northern Thai population by using epoxy resin injection technique. J. Med. Assoc. Thai., 97(12):1352-7, 2014.

Williams, P. L. Gray's Anatomy. 38 ed. Tomo I. Madrid, Harcourt Brace, 1998.

Zancolli, E. \& Cozzi, E. Atlas de Anatomía Quirúrgica de la Mano. Madrid, Médica Panamericana, 1993.

Zheng, D. W.; Li, Z. C.; Shi, R. J.; Sun, F.; Xu, L. \& Shou, K. S. Thumb reconstruction via a pedicled flap based on the superficial palmar branch of the radial artery from the wrist crease area.J. Plast. Reconstr. Aesthet. Surg., 68(11):1581-7, 2015

Dirección para correspondencia:

Dr. Nicolás E. Ottone.

Profesor Asociado

Laboratorio de Plastinación y Técnicas Anatómicas

Centro de Investigación en Ciencias Odontológicas (CICO)

Facultad de Odontología

Universidad de La Frontera

Temuco - CHILE

Recibido : 16-01-2021

Aceptado: 22-03-2021

E-mail: nicolas.ottone@ufrontera.cl 